\title{
Metástasis de adenocarcinoma de recto al conducto auditivo externo: Presentación de un caso y revisión de la literatura
}

\author{
Metastatic rectal adenocarcinoma to the external auditory canal: \\ Case report and literature review
}

Analía Elguezabal $\mathbf{R}^{1}$, Antoni Raventós $\mathbf{E}^{1}$, Julieta Landeyro S $^{1}$, Míriam Gené $\mathbf{H}^{\mathbf{1}}$, Joan J Sirvent $\mathbf{C}^{\mathbf{1}}$.

\begin{abstract}
RESUMEN
Las metástasis de neoplasias primarias de diferentes localizaciones a cabeza y cuello son un hecho infrecuente. La localización más habitual de recepción de dichas metástasis es el hueso temporal, sitio que usualmente es afectado por neoplasias que se diseminan a los huesos en general. Sin embargo, las neoplasias al conducto auditivo externo son muy raras, habiéndose publicado pocos casos en la literatura .

Presentamos el caso de un varón de 81 años con clínica de dolor, acúfenos y otorrea derecha. La otomicroscopía demostró una lesión polipoide en el conducto auditivo externo derecho. La biopsia objetivó una proliferación de células epiteliales atípicas que formaban luces glandulares anómalas con positividad para citoqueratina 20 y antígeno carcinoembrionario. Se diagnosticó como metástasis de adenocarcinoma de probable origen colónico y posteriormente se conocieron antecedentes de colectomía 9 años antes por adenocarcinoma de recto-sigma.

Las metástasis de adenocarcinoma de colon al conducto auditivo externo son un hecho infrecuente y de aparición tardía. Requieren un diagnóstico diferencial con metástasis de adenocarcinomas de otras localizaciones y, en menor medida debido a su baja frecuencia, con tumores primarios. Entre estos últimos, los que plantean mayores dificultades diagnósticas son los adenocarcinomas ceruminosos.
\end{abstract}

Palabras clave: Metástasis de colon, conducto auditivo externo, tumores oído.

\section{ABSTRACT}

Metastasis to the head and neck region are unusual. The most common site of receiving those metastasis is the temporal bone, a site which is usually affected by tumors that spread to the bones. However, metastasis to the external auditory canal are

Médico de Anatomía Patológica, Hospital Universitario Joan XXIII. Tarragona, España. 
infrequent. Only a few cases have been published in the literature. We report the case of a 81 year-old man with pain, tinnitus and right otorrhea. The otomicroscopy showed a polypoid lesion in the right ear canal. The biopsy showed an atypical proliferation of epitelial cells forming anomalous glands, which were positive for citoqueratine 20 and carcinoembryonic antigen. He was diagnosed with metastatic adenocarcinoma of colonrectum nine years previously.

Metastasis of colon adenocarcinomas to the external auditory canal are infrequent. Require a defferential diagnosis with metastatic adenocarcinomas of others locations but is always necessary to rule out primary tumors. Among these, the most challenging diagnostic is with ceruminous adenocarcinomas.

Key words: Colon metastases. External auditory canal. Ear tumors.

\section{INTRODUCCIÓN}

Las metástasis al oído son un hecho infrecuente ${ }^{1,2}$. Dentro de la región de cabeza y cuello, la localización más habitual de recepción de metástasis es el hueso temporal, siendo el conducto auditivo externo un área de muy rara afectación. Suelen recibir metástasis de tumores que normalmente se diseminan a hueso, como tumores primarios de mama, riñón y pulmón ${ }^{1}$. Los adenocarcinomas de colon se diseminan con mayor frecuencia a hígado, pulmón, peritoneo, hueso, ovarios y glándulas adrenales, existiendo pocos casos en la literatura con metástasis al oído $0^{3-5}$. Presentamos el caso de un hombre de 81 años con metástasis en conducto auditivo externo de un adenocarcinoma de recto-sigma que se había diagnosticado y tratado 9 años antes.

\section{CASO CLÍNICO}

Varón de 81 años con antecedentes patológicos de hipertensión arterial y úlcera péptica. Se presentó a la consulta de otorrinolaringología en octubre de 2008 con clínica de dolor, acúfenos y otorrea derecha de 10 días de evolución. Refirió cuadros de otitis derecha en varias ocasiones en el último año. Se realizó otomicroscopía que objetivó la presencia de un pólipo que ocupaba todo el conducto auditivo externo derecho. Se tomaron muestras para biopsia y se realizó una resonancia magnética nuclear. Esta última mostró una lesión de aspecto neoplásico que afectaba todo el conducto auditivo externo, tapizándolo circunferencialmente hasta la zona del pabellón auricular.
Dicha lesión se extendía hasta el oído medio, ocupando la práctica totalidad de la caja timpánica, pero sin observarse signos de erosión ósea sugestiva de infiltración (Figura 1). El estudio microscópico de la lesión mostraba una proliferación de células epiteliales atípicas que formaban luces glandulares anómalas. Las células tenían núcleos ovales grandes, hipercromáticos, con desproporción del núcleo-citoplasma y con una disposición en empalizada (Figura 2). El estudio inmunohistoquímico demostró positividad de membrana y

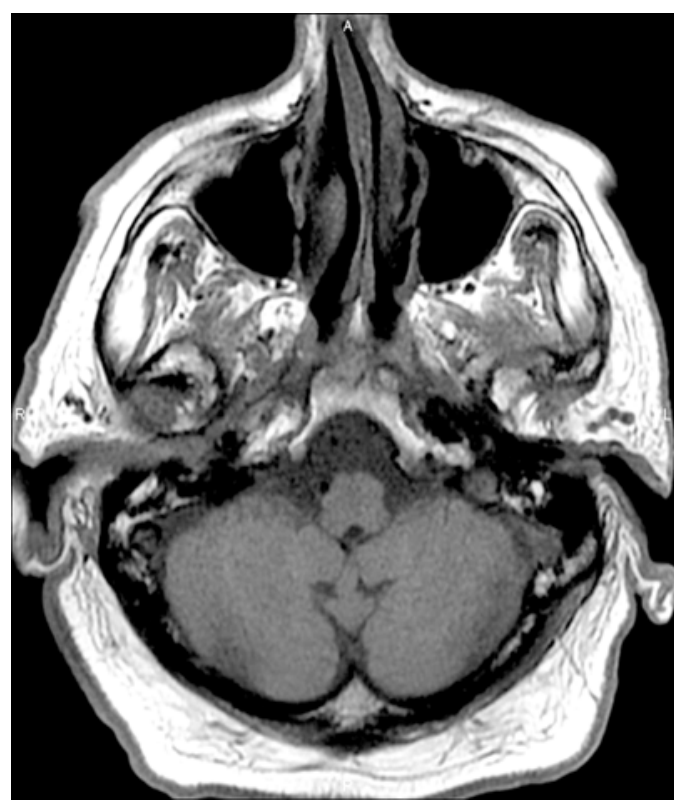

Figura 1. RMN. Ocupación del conducto auditivo externo derecho por lesión de aspecto neoplásico que infiltra la caja timpánica. 


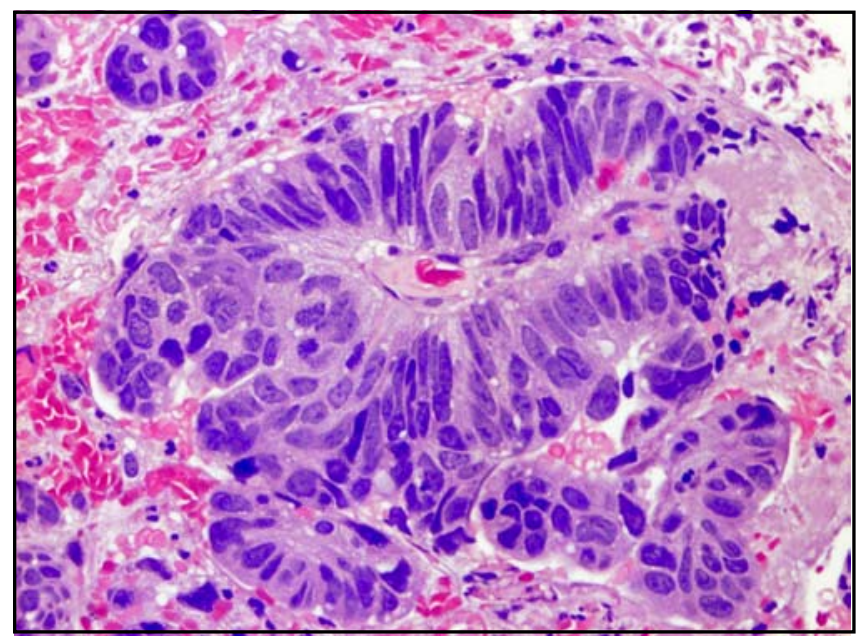

Figura 2. HE 200x. Glándulas anómalas con atipia citológica y disposición en empalizada.

citoplasmática de dichas células para citoqueratina 20 (Figura 3) y en el borde apical para antígeno carcinoembrionario, mientras que resultó negativo para citoqueratina 7. Todo esto apoyó el diagnóstico de metástasis de adenocarcinoma de probable origen colónico. Posteriormente se conocieron antecedentes de colectomía 9 años antes por adeno- carcinoma de recto-sigma (pT3, pN1) y desde hacía dos años también presentaba metástasis hepáticas. Se revisó la pieza de colectomía y se constató que la tumoración era histológica e inmunohistoquímicamente similar a la lesión descrita en el oído, confirmando el diagnóstico de metástasis de adenocarcinoma de recto-sigma.

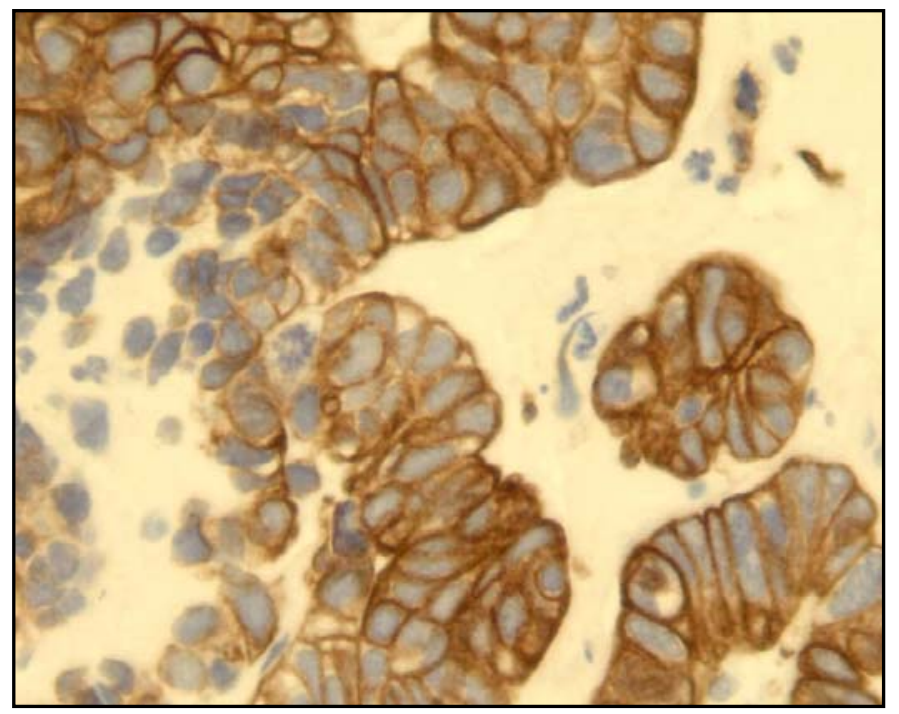

Figura 3. Positividad glandular para citoqueratina 20. 


\section{DISCUSIÓN}

Las metástasis al conducto auditivo externo son muy raras, encontrándose pocos casos en la literatura, los cuales son generalmente motivo de publicación de un solo caso. Existen además, registros de casos de metástasis al oído medio e interno provenientes de sitios distantes ${ }^{1,6}$. Los tumores de colon y recto metastatizan con mayor frecuencia a hígado, pulmón, peritoneo, hueso, ovarios y glándulas adrenales. Las metástasis cerebrales son inusuales y habitualmente signo de enfermedad terminal. También se han descrito metástasis en sitios raros como páncreas, bazo, laringe y lengua ${ }^{3-}$ 5. Con respecto al oído, en los últimos 25 años aparecen cuatro casos en la literatura, uno de ellos muy reciente, publicado en el año $2008^{7}$. Es bastante frecuente encontrar que estas metástasis aparezcan bastante tiempo después del primer tratamiento, al igual que ocurrió en nuestro caso. En 2007 hay publicado un caso de hepatocarcinoma que metastatiza en conducto auditivo ${ }^{8}$. También se recoge un caso de próstata publicado en el año $2003^{9}$ y otro de mama en el año $1994^{10}$.

La vía de diseminación que se preconiza es hemática, a través de un camino poco claro en el que intervendría el paso de las células desde el hígado, por el filtro pulmonar hacía la circulación general (puede explicar el hecho que los pacientes tengan metástasis hepáticas y no pulmonares). Otra posibilidad es desde la médula ósea del temporal y desde allí, bien por un crecimiento rápido, o lo que suele ser más frecuente, por un crecimiento muy lento aparezca la metástasis años después del tratamiento del tumor primario. Por último, otro posible mecanismo podría consistir en que las células tumorales desde la circulación linfática lleguen a la circulación vascular y desde aquí al oído medio ${ }^{1,4}$.

Clínicamente, tanto los tumores primarios de oído como las metástasis, se presentan con otorrea persistente, otalgia y/u otorragia. El diagnóstico suele ser tardío debido a la baja incidencia de tumores en esta localización y a que presentan síntomas similares a otras patologías benignas del oído. Cuando la enfermedad se halla avanzada, pueden encontrarse parálisis facial periférica, hipoacusia 0 adenopatías cervicofaciales $1,6,7,11,12$. La técnica de imagen utilizada para evaluar la localización, extensión ósea y a órganos vecinos es la tomografía axial computarizada (TAC), reservándose el uso de la RMN para la evaluación de lesiones de partes blandas ${ }^{6,11,12}$. El diagnóstico de certeza se realiza con la confirmación histológica. Se requiere una pieza adecuada para demostrar si existe o no infiltración a cartílago, hueso, vasos sanguíneos 0 perineural, datos que habitualmente se objetivan mejor en la periferia del tumor ${ }^{6,11,12}$.

El diagnóstico diferencial se realiza principalmente con lesiones metastásicas provenientes de sitios distantes, para lo cual es indispensable conocer los antecedentes patológicos del paciente, estudios de extensión y analizar las características histológicas de la lesión. Estas últimas, sumadas al uso de técnicas de inmunohistoquímica, pueden ayudar al diagnóstico orientativo 0 de certeza del origen de la metástasis. En menor medida hay que tener en cuenta a los tumores primarios del conducto auditivo externo. Las variantes malignas de esta localización son muy infrecuentes, afectando a 1-2 personas por cada millón de habitantes con una edad media de 55 años y sin predominio de sexos $^{6}$. El carcinoma epidermoide constituye el tumor más frecuente en esta localización. A pesar de su baja frecuencia, su incidencia es un poco más alta en pacientes con enfermedades otológicas previas, como infecciones crónicas recurrentes y también se lo ha asociado con exposición a radiaciones o productos químicos. La supervivencia depende del estadio y el tratamiento aplicado ( $90 \%$ para estadios I y II y $80 \%-35 \%$ para estadios III y IV). Constituyen factores pronósticos la presencia de ganglios linfáticos positivos, la parálisis facial y la extensión intracraneal ${ }^{6,11,13}$. Sin embargo el diagnóstico diferencial más importante cuando se trata de un tumor de estirpe glandular, como es en nuestro caso, es con los tumores ceruminosos primarios de esta localización. La incidencia y comportamiento de estos tumores continúa siendo poco clara debido a la confusa terminología, clasificación y definiciones histológicas. Existen diferentes tipos de tumores de glándulas ceruminosas del conducto auditivo externo agrupadas bajo el término de ceruminoma, entre las que se incluyen los siguientes: adenoma 
ceruminoso, adenoma pleomórfico de glándulas ceruminosas, adenocarcinoma de bajo y alto grado ceruminoso, carcinoma mucoepidermoide ceruminoso, carcinoma adenoide quístico de glándulas ceruminosas ${ }^{6,11-13}$. El carcinoma adenoide quístico constituye la entidad más frecuente dentro de este grupo. Presenta las mismas características histológicas que los hallados en glándulas salivales y pueden presentar metástasis pulmonares incluso años después del diagnóstico inicial ${ }^{6,11-13}$. Sin embargo, histológicamente, son los adenocarcinomas de bajo y alto grado los que plantean una dificultad diagnóstica con las metástasis provenientes de colon y recto. Los de bajo grado se caracterizan por presentar glándulas ceruminosas normales retenidas, leve pleomorfismo nuclear y escasas figuras de mitosis. A veces, son difíciles de diferenciar de los adenomas ceruminosos, para lo cual se requiere la confirmación histológica de infiltración tumoral, por lo que suelen precisarse resecciones quirúrgicas amplias. Los de alto grado, en cambio, invaden extensamente los tejidos vecinos, presentan infiltración en sábana por glándulas irregulares con gran pleomorfismo nuclear y abundantes figuras de mitosis ${ }^{11,12}$. La utilización de técnicas de inmunohistoquímica ayudarían al diagnóstico, apoyando el origen colónico si se demuestra positividad para citoqueratina 20 y negatividad para citoqueratina 7 .

El tratamiento de las metástasis depende del estado del paciente. Si la metástasis fuese única podría realizarse excisión quirúrgica, pero usualmente son pacientes que presentan una enfermedad ampliamente diseminada. Esto plantea la necesidad de otros tratamientos, como pueden ser la radioterapia $y / 0$ quimioterapia, aunque hay que tener en cuenta que no están exentos de efectos adversos $\mathrm{s}^{6,11,12}$. En nuestro paciente se decidió una actitud expectante debido a la edad avanzada y a su buen estado general, con posibilidad de aplicar un tratamiento paliativo cuando fuera necesario. Los tumores primarios suelen tratarse mediante cirugía acompañada de quimioterapia previa o posterior, pero como ya se ha dicho, el pronóstico depende del estadio y hay que tener en cuenta que, en muchos casos la lesión no está localizada en el momento del diagnóstico.

\section{BIBLIOGRAFÍA}

1. Carson hJ, Krivit JS, Ellers SG, Ellers SG. Metastasis of colonic adenocarcinoma to the external ear canal: An unusual case with a complex pattern of disease progression. Ear Nose Throat J 2005; 84: 36-8.

2. Michaelson, PG, Lowry TR. Metastatic renal cell carcinoma presenting in the external auditory canal. Otolaryngol Head Neck Surg 2005; 133 : 979-80.

3. Danikas D, Theodorou S, Arvanitis M, Zinterhofer L, Rienzo A. Malar metastasis from rectal carcinoma: A case report. Am Surg 1999; 65: 1150-2.

4. Mason AC, Azari KK, Farkas lM, Duvvuri U, MYERS EN. Metastatic adenocarcinoma of the colon presenting as a mass in the mandible. Head Neck 2005; 27: 729-32.

5. Bin Sabir Husin Athar PP, bte Ahmad Norhan N, bin Saim L, bin Md Rose I, bte Ramli R. Metastasis to the sinonasal tract from colon adenocarcinoma. Ann Acad Med Singapore 2008; 37: 788-93.

6. Rodriguez Paramas A, Gil Carrasco R, Arena Britez 0, Scola YuRRIta B. Tumores malignos del conducto auditivo externo y oído medio. Acta Otorinolaringol Esp 2004; 55: 470-4.

7. Carr S, Anderson C. Metasttic rectal adenocarcinoma in the external auditory canal. $J$ Laryngol Otol 2008; 4: 1-2.

8. Yамамото T, Komune $S$. Metastatic hepatocellular carcinoma of the extenal auditory canal. World J Gastroenterol 2007; 13: 6436-8.

9. Ueyama H, Kumamoto T, Narusako T, Fujimoto $\mathrm{S}$, Goda M, Isono M, Kobayashi H, Tsuda T. Solitary metastasis of prostatic cancer to the internal auditory canal. Clin Neurol Neurosurg 2003; 105: 180-2.

10. Cumberworth VL, Friedmann I, Glover GW. Late metastasis of breast carcinoma to the external auditory canal. J Laryngol Otol 1994; 108: 80810.

11. Soon LS, Bullok M, Prince ME. Ceruminous adenocarcinoma: A rare tumor of the external auditory canal. J Otolaryngol 2001; 30: 373-7. 
12. Tzagaroulakis a, Pasxalidis J, Papadimitriou N, Boussiotou A, Nikolopoulos T, Korres S, et al. Recurrent ceruminous adenocarcinoma of the external auditory canal. ORL $J$ Otorhinolaryngol Relat Spec 2003; 65: 300-2.
13. Elsürer C, Senkal Ha, Zayyan E, Yilmaz T, Kaya S. Bilateral external auditory canal squamous cell carcinoma: a case report. Eur Arch Otorhinolaryngol 2007; 264: 941-5.

Dirección: Dr Mallafré Guash, 4

Código postal: 43007, Tarragona. España

E mail: analiael@yahoo.com 